\title{
Le projet de nouvelles recommandations de la CIPR : une approche élargie de l'optimisation de la radioprotection
}

\author{
J. LOCHARD ${ }^{1}$
}

(Manuscrit reçu le 25 janvier 2005, accepté le 25 juillet 2005)

RÉSUMÉ Dans le cadre de la préparation de ses nouvelles recommandations, la CIPR a développé un texte entièrement consacré au principe d'optimisation de la protection. Ce texte, qui s'inscrit dans la lignée des publications antérieures de la Commission sur le sujet (Publications 37 et 45) rappelle la nécessité d'adopter une approche pragmatique en faisant appel aux techniques quantitatives quand elles sont pertinentes tout en gardant à l'esprit que le savoir-faire et l'expérience sont souvent suffisants pour assurer une protection de qualité. Enfin, il vise essentiellement à adapter la démarche de l'optimisation aux évolutions récentes en matière de gestion du risque, qui mettent l'accent de plus en plus sur l'implication directe des acteurs concernés dans les processus de préparation des décisions.

ABSTRACT The new ICRP recommendations' project: A broader approach of the optimisation of radiation protection.

In the framework of the preparation of its new recommendations ICRP has developed a new text on the optimisation of radiaological protection. This text prolongs the previous publications on the principle (Publications 37 and 45) reminding the need to adopt a pragmatic approach combining quantitative techniques when they are relevant as well as know-how and past experience which are often sufficient to ensure good protection. Moreover, it aims at adapting the optimisation process to the recent evolutions of risk management with the increasing role of stakeholder involvement in the decision framing.

Keywords: ICRP / Recommendations / Radiological protection / Optimisation

\section{Introduction}

La CIPR a mis en consultation sur son site Internet son projet de nouvelles recommandations (RP05) au cours de l'été 2004. En parallèle, elle a chargé ses divers comités de préparer des textes complémentaires visant à éclairer différents aspects de son projet, qui devraient être publiés en même temps que les recommandations. Parmi ces textes l'un est entièrement consacré au principe d'optimisation de la protection qui reste un des piliers fondamentaux du système de protection radiologique. Ce nouveau texte s'inscrit dans la lignée des

1 CEPN, route du Panorama, B.P. 48, 92263 Fontenay-aux-Roses Cedex, France. 
publications antérieures de la CIPR sur le sujet (Publication 37 de la CIPR, 1983 ; publication 45 de la CIPR, 1986) et vise essentiellement à adapter la démarche de l'optimisation aux évolutions récentes en matière de gestion du risque, qui mettent l'accent de plus en plus sur l'implication directe des acteurs concernés dans les processus de préparation des décisions. Il apporte également un éclairage utile sur l'articulation du principe d'optimisation avec les autres principes et concepts de base du nouveau système de protection et en particulier la contrainte de dose individuelle.

Les paragraphes qui suivent présentent de façon synthétique les aspects les plus importants de la future publication de la CIPR sur l'optimisation de la radioprotection qui est consultable sur le site Internet de la Commission et ouverte à commentaires.

\section{L'histoire du principe d'optimisation de la radioprotection}

La formulation du principe d'optimisation a évolué au cours du temps. Les fondements de ce principe apparaissent dès 1950 lorsque la CIPR, pour tenir compte de l'incertitude associée à l'apparition des effets stochastiques, recommande que « tous les efforts soient entrepris pour réduire les expositions à tous les types de rayonnements ionisants au niveau le plus bas possible ». Progressivement, la formulation du principe a évolué dans le souci d'allier la mise en œuvre de politiques de réduction des expositions et le maintien de la viabilité des activités, ce qui a conduit à intégrer des considérations économiques et sociales dans l'évaluation des actions de réduction des expositions. C'est en 1977, dans la publication 26 de la CIPR (ICRP, 1977), que la formulation actuelle est adoptée : «maintenir les expositions aussi bas que raisonnablement possible compte tenu des facteurs économiques et sociaux $»$.

L'approche retenue pour la mise en œuvre pratique de ce principe a également progressivement évoluée. En 1973, la publication 22 de la CIPR (ICRP, 1973) introduit l'analyse coût-bénéfice pour la sélection des options de radioprotection. Il est ensuite apparu nécessaire de ne pas se restreindre au seul critère économique et la publication 55 en 1988 propose « la procédure d'optimisation » qui insiste sur la nécessité d'introduire d'autres facteurs dans le processus décisionnel. Les publications 73,77 et $81(1996,1997,1998)$ renforcent la nécessité d'aller au-delà des approches quantitatives et de considérer les aspects managériaux ainsi que les dimensions sociales et éthiques. Enfin, un pas supplémentaire important vers l'ouverture du processus est accompli avec la publication 82 (1999) qui recommande d'impliquer les parties prenantes dans le processus décisionnel. 


\section{Le processus d'optimisation}

\subsection{Rôle du principe d'optimisation}

Le principe d'optimisation vient compléter le premier principe qui requiert des restrictions de dose individuelle (contraintes de dose, limites de dose) comme niveau de base de la protection. La nécessité de réduire les expositions en dessous des contraintes de dose provient de l'hypothèse qu'il existe une probabilité de développer des effets sanitaires même pour de faibles augmentations de l'exposition aux rayonnements. Ces contraintes constituent ainsi la borne supérieure à partir de laquelle le processus d'optimisation est engagé.

Tout en conservant la formulation de la CIPR60, à savoir que « le niveau des doses individuelles, le nombre de personnes exposées et la probabilité de recevoir des expositions quand celles-ci ne sont pas certaines, devraient être maintenus aussi bas que raisonnablement possible en tenant compte des facteurs économiques et sociaux », le RP05 souligne que le processus est plus large. Il nécessite en effet l'adoption d'une «culture de sûreté et de radioprotection », intégrant une palette d'approches quantitatives et qualitatives. Cette culture de sûreté et de radioprotection devrait ainsi amener chaque responsable de la maîtrise des expositions à se poser la question « ai-je fait tout ce qui est raisonnable pour éviter ou réduire les doses compte tenu de la situation ? ». Pour la CIPR, la réponse à cette question est une affaire de «jugement » et nécessite une coopération entre les parties prenantes.

\subsection{La définition du cadre dans lequel s'inscrit le processus d'optimisation}

La première étape de tout processus d'optimisation consiste à analyser la situation d'exposition afin d'identifier les caractéristiques qui lui sont propres et qui vont être prises en considération dans le processus décisionnel.

Le projet de publication sur l'optimisation identifie ainsi une liste (nonexhaustive) des particularités d'une situation d'exposition, tenant compte :

- des caractéristiques de la population exposée (sexe, âge, modes de vie...),

- des caractéristiques des expositions (distribution dans le temps et dans l'espace, nombre de personnes exposées, doses individuelles maximales, minimales et moyennes, expositions potentielles...),

- des transferts d'exposition entre groupes,

- des considérations et des valeurs sociales (équité, capacité à contrôler, considérations intergénérationnelles, bénéfice individuel, bénéfice social...), 
- des considérations environnementales,

- des considérations économiques et techniques.

\subsection{Les caractéristiques du processus d'optimisation}

Le processus d'optimisation est un processus continu et itératif destiné à prévenir les expositions avant qu'elles ne surviennent. L'évaluation et la sélection des options de protection identifiées peuvent être basées aussi bien sur des approches quantitatives que qualitatives. La diversité des facteurs à prendre en compte implique souvent la nécessité de faire appel à un jugement qualitatif pour prendre les décisions. Dans cette perspective, l'implication des parties prenantes dans le processus d'aide à la décision se révèle de plus en plus souvent comme étant un moyen efficace.

\subsection{L'implication des parties prenantes}

Déjà mentionné dans la publication 82 , l'implication des parties prenantes est désormais considérée comme un élément important du processus d'optimisation. L'expérience en la matière a en effet montré que c'est un moyen efficace pour intégrer les considérations et valeurs sociales dans les décisions, améliorer la qualité de ces dernières, résoudre les conflits entre des intérêts divergents, construire une compréhension partagée entre toutes les parties concernées et, enfin, renforcer la confiance dans les institutions.

La publication sur l'optimisation souligne le fait que l'implication des parties prenantes n'est pas une démarche qui doit être appliquée systématiquement mais au contraire au cas par cas en fonction des circonstances. De plus, elle précise bien que, quel que soit le degré d'implication des acteurs dans un processus décisionnel relatif à l'optimisation de la protection, la responsabilité finale en matière de décision reste la prérogative du décideur, c'est-à-dire le responsable de la source ou l'autorité.

\subsection{Le résultat du processus d'optimisation}

Ce dernier est toujours spécifique à la situation d'exposition considérée et il correspond au meilleur niveau de protection qu'il est possible d'atteindre compte tenu des circonstances. Par conséquent, il n'est pas opportun de fixer a priori des niveaux de dose en-dessous desquels l'optimisation ne devrait pas s'appliquer. En fonction de la situation d'exposition, le résultat du processus d'optimisation pourra être proche ou au contraire très en-dessous de la valeur de la contrainte de dose correspondante. 


\section{Optimisation et distribution des expositions}

Pour être pertinente, la comparaison d'options de protection pour une situation d'exposition implique souvent de prendre en compte la distribution des doses individuelles parmi les groupes de personnes exposées. Dans le passé, le recours à la dose collective pour caractériser la distribution des doses individuelles était fréquent. Si dans le cas des expositions des travailleurs une telle démarche est le plus souvent légitime, la dose collective servant alors d'indicateur de performance pour une tâche ou opération donnée dans une installation, l'utilisation du concept dans le cas des expositions du public a en revanche toujours été beaucoup plus problématique. En particulier lorsque les expositions concernent de très grandes populations sur des espaces très étendus et pendant des périodes de temps très longues elle conduit à une agrégation excessive de l'information qui masque les caractéristiques de la population exposée et des expositions correspondantes.

Pour pallier cet inconvénient, la CIPR suggère dorénavant d'estimer les doses individuelles pour des groupes de population définis en fonction des caractéristiques des individus exposés et de leur exposition. Ainsi, à partir d'une interrogation sur quand, où et par qui les doses individuelles sont reçues, il est possible de construire une «matrice de doses » qui permet alors de regrouper, d'une façon pertinente pour la radioprotection, des sous-groupes d'individus exposés en fonction de leurs caractéristiques (sexe, âge, mode de vie, groupe sensible...) mais aussi de celles de la situation d'exposition en termes de temps (court, moyen, long terme) et de lieu (local, régional, national...), afin de choisir les actions de protection les mieux adaptées. Ce faisant, il est possible de prendre en considération les préférences et les valeurs des parties impliquées dans le processus de décision, en accordant plus ou moins d'importance à tel ou tel paramètre.

\section{L'application de l'optimisation par les opérateurs et les autorités}

Dans la dernière section de la publication consacrée à l'application du principe d'optimisation la CIPR souligne clairement que le choix et la mise en œuvre pratique des actions de protection relève de la responsabilité des opérateurs, les autorités ayant celle d'exiger et de contrôler l'application du principe. Elle considère par ailleurs que le développement d'une culture de protection au sein des autorités comme des opérateurs est un facteur déterminant pour une mise en œuvre effective du principe. Elle précise enfin que l'optimisation est plus une obligation de moyens que de résultats et que l'existence d'un dialogue de qualité entre les opérateurs et les autorités est un gage de succès pour une application efficace du principe d'optimisation. 


\section{Conclusion}

Le texte sur l'optimisation proposé par la CIPR en complément de ses nouvelles recommandations est essentiellement conceptuel. Il est complété par une série d'annexes qui illustrent différents domaines d'application de l'optimisation de la radioprotection : les expositions professionnelles, les expositions du public dues aux installations nucléaires, les expositions au radon dans les habitations, et qui fournissent par ailleurs des références de base pour un lecteur soucieux de rentrer dans les détails de l'application du principe. Ce dernier est réaffirmé à tous les niveaux comme le cœur du système de radioprotection sur le plan de l'action.

En conclusion, ce nouveau texte met surtout en évidence la nécessité de tenir compte du contexte particulier à chaque situation d'exposition pour une application efficace du principe d'optimisation. Il rappelle, de manière récurrente, la nécessité d'adopter une approche pragmatique en faisant appel aux techniques quantitatives quand elles sont pertinentes tout en gardant toujours à l'esprit que le savoir-faire et l'expérience sont souvent suffisants pour assurer une protection de qualité. Enfin, il souligne que l'implication des parties prenantes dans le processus de préparation de la décision fait désormais partie du processus d'optimisation au même titre que les méthodes classiques d'aide à la décision.

\section{RÉFÉRENCES}

ICRP Publication 22 (1973) Implication of Commission Recommendations that Doses be Kept as Low as Readily Achievable. Pergamon Press, Oxford.

ICRP Publication 26 (1977) Recommendations of the International Commission on Radiological Protection, Ann. ICRP 1(3). Superseded by ICRP Publication 60.

ICRP Publication 37 (1983) Cost-Nenefit Analysis in the Optimization of Radiation Protection, Ann. ICRP 10(2/3).

ICRP Publication 45 (1986) Quantitative Bases for Developing a Unified Index of Harm, Ann. ICRP 15(3).

ICRP Publication 55 (1988) Optimization and decision-making in Radiological Protection, Ann. ICRP $20(1)$.

ICRP Publication 73 (1996) Radiological Protection and Safety in Medicine, Ann. ICRP 26(2).

ICRP Publication 77 (1997) Radiological Protection Policy for the Disposal of Radioactive Waste, Ann. ICRP 27(suppl.).

ICRP Publication 81 (1998) Radiation Protection Recommendations as Applied to Disposal of LongLived Solid Radioactive Waste, Ann. ICRP 28(4).

ICRP Publication 82 (1999) Protection of the public in Situations of Prolongated Radiation Exposure, Ann. ICRP 29(1/2). 